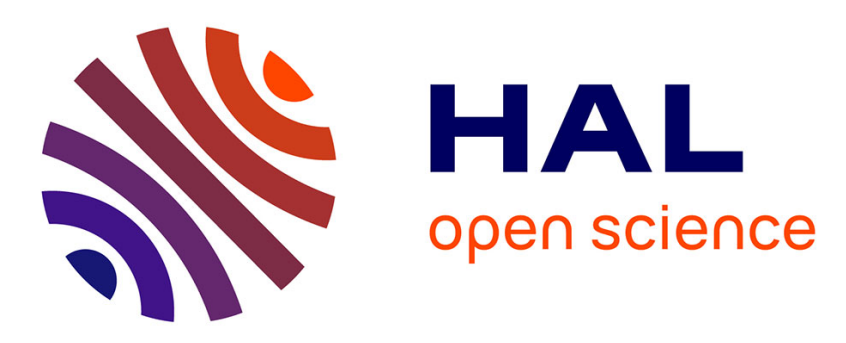

\title{
Periodization of random media and representative volume element size for linear composites
}

\author{
Karam Sab, Boumediene Nedjar
}

\section{To cite this version:}

Karam Sab, Boumediene Nedjar. Periodization of random media and representative volume element size for linear composites. Comptes rendus de l'Académie des sciences. Série IIb, Mécanique, 2005, 333, pp.187-195. 10.1016/j.crme.2004.10.003 . hal-00121487

\section{HAL Id: hal-00121487 \\ https://hal.science/hal-00121487}

Submitted on 13 Apr 2007

HAL is a multi-disciplinary open access archive for the deposit and dissemination of scientific research documents, whether they are published or not. The documents may come from teaching and research institutions in France or abroad, or from public or private research centers.
L'archive ouverte pluridisciplinaire HAL, est destinée au dépôt et à la diffusion de documents scientifiques de niveau recherche, publiés ou non, émanant des établissements d'enseignement et de recherche français ou étrangers, des laboratoires publics ou privés. 


\title{
Periodization of random media and representative volume element size for linear composites.
}

\author{
Karam Sab and Boumediene Nedjar \\ Institut Navier, \\ LAMI, Ecole Nationale des Ponts et Chaussées. \\ 6-8 avenue Blaise Pascal, Champs-sur-Marne, \\ 77455 Marne-la-Vallée cedex 2, France. \\ E-mail address: sab@lami.enpc.fr
}

June 21, 2004

\begin{abstract}
Several existing numerical studies show that the effective linear properties of random composites can be accurately estimated using small volumes subjected to periodic boundary conditions - more suitable than homogeneous strain or stress boundary conditions- providing that a sufficient number of realizations are considered. Introducing the concept of periodization of random media, this Note gives a new definition of representative volume element which leads to estimates of its minimum size in agreement with existing theoretical results. A qualitative convergence criterion for the numerical simulations is proposed and illustrated with finite element computations.

Résumé: Périodisation des milieux aléatoires et détermination de la taille du volume élémentaire représentatif des composites linéaires. Plusieurs simulations numériques montrent que les propriétés effectives linéaires des matériaux aléatoires peuvent être calculées sur de petits échantillons soumis à des conditions limites périodiques - plus adaptées que les conditions uniformes en contrainte ou en déformation pourvu que le nombre d'échantillons considérés soit suffisamment grand. En introduisant le concept de périodisation des milieux aléatoires, cette Note donne une nouvelle définition du volume élémentaire représentatif qui conduit à des estimations de sa taille minimale conformes aux résultats théoriques existants. Un critère qualitatif de convergence des simulations numériques est proposé et illustré par des calculs par éléments finis.

Keywords: Homogenization; Periodization; Random media; Representative volume element.

Mots-clés: Homogénéisation; Périodisation; Milieux aléatoires; Volume élémentaire représentatif.
\end{abstract}




\section{Version française abrégée}

On observe dans les simulations numériques que la taille du volume élémentaire représentatif (VER) d'un milieu aléatoire linéaire peut être petite à condition d'utiliser des conditions limites périodiques et de faire la moyenne sur beaucoup d'échantillons [1], [2], [3]. L'objet de cette Note est d'expliquer cette observation et de faire le lien avec les estimations approchées de la taille du VER données dans [4].

On utilise pour cela le cadre théorique adapté à l'homogénéisation des milieux aléatoires [5], [6]. Le milieu infini est décrit par un espace de probabilité $(\Omega, \mathcal{A}, \mathbb{P})$ où $\Omega$ représente l'ensemble des réalisations possibles $\omega \in \Omega . \tau_{\mathbf{t}} \omega$ est la réalisation obtenue lors d'une translation $\mathbf{t}$. On suppose que $\tau_{\mathbf{t}}$ a les propriétés (1) et (2) qui assurent l'homogénéité statistique du milieu. A chaque variable aléatoire réelle $X: \Omega \rightarrow \mathbb{R}$ est associé le processus statistiquement homogène (s.h.) $\widetilde{X}$ défini par (3). On définit dans (4) la fonction de corrélation de deux processus s.h. de carré intégrable. Ici $\mathbb{E}$ est l'espérance mathématique. L'élasticité du milieu est décrite par $\widetilde{\mathbf{c}}(\omega, \mathbf{t})$, le processus s.h. des tenseurs de rigidité. Pour un bi-phasé $\mathbf{c}(\omega)=\chi(\omega) \mathbf{c}^{I}+(1-\chi(\omega)) \mathbf{c}^{I I}$ où la fonction caractéristique $\widetilde{\chi}(\omega, \mathbf{t})=\chi\left(\tau_{-\mathbf{t}} \omega\right)$ de la phase $I$ vaut 1 si $\mathbf{t}$ est dans la phase $I$, et 0 sinon. $S_{k}$ est la probabilité (5) de trouver $\mathbf{t}_{1}, \cdots, \mathbf{t}_{k}$ dans la phase $I$ . A titre d'exemple, on considère des inclusions sphériques de rayon $R$ (phase $I I$ ) aléatoirement distribuées dans une matrice (phase $I$ ) selon le processus de Poisson, avec interpénétration possible. Alors, $\omega=\left\{\mathbf{x}_{n}, n \in \mathbb{N}\right\}$ est l'ensemble des centres des inclusions, $\tau_{\mathbf{t}} \omega=\left\{\mathbf{x}_{n}+\mathbf{t}, n \in \mathbb{N}\right\}, \chi(\omega)=1$ si $\operatorname{Inf}_{n}\left|\mathbf{x}_{n}\right|>R$, et $=0$ sinon, et $S_{k}$ est donné par (6) où $v_{k, R}\left(\mathbf{t}_{1}, \cdots, \mathbf{t}_{k}\right)$ est le volume du domaine obtenu par la réunion des sphères de rayon $R$ centrées en $\mathbf{t}_{1}, \cdots, \mathbf{t}_{k}$, et $\rho>0$ est le nombre moyen d'inclusions par unité de volume [7], [8].

La détermination du tenseur d'élasticité homogénéisé $\mathbf{C}^{\text {hom }}$ se fait par la résolution du problème auxiliaire (7-8). En introduisant un milieu de comparaison homogène, $\mathbf{C}^{0}$, le tenseur de polarisation $\mathbf{p}=\boldsymbol{\sigma}-\mathbf{C}^{0}:\left(\mathbf{e}^{*}+\mathbf{E}\right)$ est solution de (9) et rend stationnaire la fonctionnelle (11). Voir [9]. Ici, l'opérateur $\boldsymbol{\Gamma}^{0}$ est défini par (10). Il s'exprime en fonction du noyau de Green du milieu de comparaison lorsque les corrélations $\left\langle c_{i j m n} p_{k l}\right\rangle$ (t) sont dans $L^{2}\left(\mathbb{R}^{d}\right)$. Dans ce cas, $\mathbf{C}^{\text {hom }}$ est aussi donné par (12-13). En fait, $-\mathbf{C}^{0}: \mathbf{e}^{*}$ peut remplacer $\delta \mathbf{p}$ dans (12-13) de manière équivalente.

On se restreint dans la suite aux matériaux bi-phasés. L'idée de la périodisation est de construire une suite $\left(\Omega^{L}, \mathcal{A}^{L}, \mathbb{P}^{L}\right)_{L>0}$ d'espaces de probabilité statistiquement homogènes sur le sous-ensemble $\Omega^{L} \subset \Omega$ des réalisations $L$ périodiques (14) telle que (15). Ici $\left\{\mathbf{e}_{1}, \cdots, \mathbf{e}_{d}\right\}$ est la base canonique de $\mathbb{R}^{d}$. Le tenseur effectif $\mathbf{C}^{\text {hom, } L}$ relatif au milieu périodisé est donné par (16), où l'opérateur $\boldsymbol{\Gamma}^{0, L}$ est celui de l'homogénéisation périodique classique sur la cellule de base cubique de taille $L, V^{L}$. Une analyse de Fourier [11] montre que cet opérateur a la propriété (18) où $\left\langle c_{i j m n} p_{k l}^{L}\right\rangle^{\infty}$ est défini par (17). Comparant les équations (12-13) et (16-18), on s'attend à (19) et à la convergence de $\mathbf{C}^{\text {hom, } L}$ vers $\mathbf{C}^{\text {hom }}$ pour $L$ croissant. On dit que $V^{L}$ est un $\epsilon$-VER si (20) est vraie. Ici $\|\cdot\|$ est une norme sur $\mathbb{R}^{d} \times \mathbb{R}^{d}$. En effet, pour une périodisation idéale telle 
que $\mathbb{E}^{L} \mathbf{c}=\mathbb{E} \mathbf{c}$ et $\left\langle c_{i j m n} p_{k l}^{L}\right\rangle^{\infty}(\mathbf{t})=\left\langle c_{i j m n} p_{k l}\right\rangle(\mathbf{t}), \forall \mathbf{t} \in V^{L}, \epsilon$ serait l'erreur relative entre $\mathbf{C}^{\text {hom, } L}$ et $\mathbf{C}^{\text {hom }}$. Les périodisations couramment utilisées ne sont pas idéales au sens précédent et introduisent une erreur supplémentaire. Donc, pour une erreur relative $\epsilon$ donnée, il faut généralement adopter dans les calculs une cellule de base de taille supérieure à celle du $\epsilon$-VER.

On sait d'après [10] que $\left\langle c_{i j m n} p_{k l}\right\rangle$ est fonction de $\mathbf{C}^{0}, \mathbf{c}^{I}, \mathbf{c}^{I I}$ et de toutes les fonctions $S_{k}$. L'approximation utilisée dans [4] consiste à se restreindre dans (11) aux tenseurs de polarisation constants par phase: $\mathbf{p}^{H S W}(\omega)=\chi(\omega) \mathbf{p}^{I}+$ $(1-\chi(\omega)) \mathbf{p}^{I I}$. Les corrélations $\left\langle c_{i j m n} p_{k l}^{H S W}\right\rangle$ s'expriment alors en fonction de $\mathbf{C}^{0}, \mathbf{c}^{I}, \mathbf{c}^{I I}, \mathbf{p}^{I}, \mathbf{p}^{I I}, S_{1}$ et $S_{2}$, et on établit à l'aide de (6) qu'elles s'annulent pour $|\mathbf{t}| \geq D=2 R$ dans le cas du bi-phasé décrit ci-dessus. D'après la définition 2 , si on se restreint à la statistique d'ordre 2 , une estimation de la taille minimale du VER est $2 D$, pour tout contraste entre les phases et tout $\epsilon$.

La périodisation du bi-phasé est comme suit: les centres des inclusions dans $V^{L}$ sont tirés selon un processus ponctuel de Poisson, puis ils sont étendus par $L$-périodicité à tout l'espace. On obtient un milieu s.h. dont chaque réalisation est $L$-périodique avec les fonctions $S_{k}^{L}$ données par (21) où l'on tient compte dans le calcul du volume $v_{k, R}^{L}$ de toutes les images par périodicité des inclusions simulées dans $V^{L}$. Les propriétés (22) sont faciles à établir.

Enfin, afin d'illustrer l'analyse théorique, une simulation par éléments finis $2 \mathrm{D}$ avec le code CESAR-LCPC (éléments Q4 longueur $/ D=\frac{1}{16} \sqrt{\frac{\pi}{2}}$ ) a été réalisée dans le cas où les deux phases sont isotropes (coefficient de Poisson 0.3 identique, contrastes 10 et 100 sur les modules de cisaillement, $S_{1}=0.5$ ). Les valeurs $L / D(1.253,2.507,5.013$ et 10.026$)$ correspondant à $16 \times 16,32 \times 32$, $64 \times 64$ et $128 \times 128$ éléments ont été considérées. Les techniques de transformée de Fourier rapide ont été utilisées pour le calcul des fonctions de corrélation. Les résultats sont synthétisés dans le Tableau 1 où $\bar{G}$ représente la moyenne du module de cisaillement normalisé, $s$ son écart-type, $n$ le nombre d'échantillons est tel que $1,96 \frac{s}{\sqrt{n} \bar{G}} \leq 0.02$. L'évaluation numérique de $S_{1}$ est aussi donnée. La figure 1 montre que la fonction de corrélation normalisée $\left\langle\chi e_{12}^{L}\right\rangle$ pour $L / D=10.026$ est négligeable en dehors d'un disque de rayon $D$. La taille minimale du VER est donc $2 D$, ce qui est en accord avec les estimations de la statistique d'ordre 2. Par ailleurs, d'après le Tableau $1 \mathrm{~b}$ et la figure 2 qui montre un profil diagonal de la figure 1 pour trois valeurs de $L / D$, on voit que les prédictions du milieu périodisé ne sont pas bonnes pour $L / D=2.507$. Elles le deviennent pour $L / D=5.013$ lorsque $\delta \mathbf{c}(0)$ et $\delta \mathbf{p}^{L}(\mathbf{t})$ ne sont plus corrélés pour $\mathbf{t}$ près du bord de $V^{L}$. Pour les forts contrastes, la convergence de $\mathbf{C}^{\text {hom, } L}$ vers $\mathbf{C}^{\text {hom }}$ a donc lieu pour une taille supérieure à la taille minimale du VER. 


\section{Introduction}

Several existing numerical studies [1], [2], [3] show that the effective linear properties of random composites can be accurately estimated using small volumes subjected to periodic boundary conditions, more suitable than homogeneous strain or stress boundary conditions, providing that a sufficient number of realizations are considered. On the other hand, using Hashin-Shtrikman-Willis (H-S-W) approximation method for the derivation of a nonlocal constitutive equation relating the ensemble averages stress and strain for an infinite random medium, estimates of the minimum representative volume element (RVE) size are provided in [4]. Introducing the concept of periodization of random media, this Note bridges the gap between these studies. In sections 2 and 3 the appropriate probabilistic framework for homogenization of random media is recalled [5], [6]. Section 4 is devoted to the periodization concept which leads to a new definition of RVE presented in section 5. Similarly to [4], the new definition requires ensemble averaging on many samples of the random medium. Finally, the theoretical analysis is illustrated in section 6 with finite element simulations of a two-phase composite.

\section{Statistically homogeneous random media}

A random infinite medium is modelled by a probability space $(\Omega, \mathcal{A}, \mathbb{P})$ where $\Omega$ is the sample space of all possible realizations, $\mathcal{A}$ is a $\sigma$-algebra and $\mathbb{P}$ is a probability measure which attributes to each subset of $A \subset \Omega$ in $\mathcal{A}, A \in \mathcal{A}$, its probability $0 \leq \mathbb{P}(A) \leq 1$ with $\mathbb{P}(\Omega)=1$. When the medium is statistically homogeneous (s.h.), two points of the physical space, identified to $\mathbb{R}^{d}$, $d=2,3$, are statistically undistinguishable. Consider a realization $\omega \in \Omega$ of the random medium and let $\tau_{\mathbf{t}} \omega$ denote the realization corresponding to the translated medium by vector $\mathbf{t}$. It is assumed that $\tau_{\mathbf{t}}$ is a measure preserving flow on $(\Omega, \mathcal{A}, \mathbb{P})$, i.e. a one-one application from $\Omega$ into itself satisfying the group property:

$$
\tau_{0}=\text { Identity }, \quad \forall \mathbf{t}, \mathbf{u} \in \mathbb{R}^{d} \tau_{\mathbf{t}} \circ \tau_{\mathbf{u}}=\tau_{\mathbf{t}+\mathbf{u}}
$$

and which preserves the probability measure:

$$
\forall \mathbf{t} \in \mathbb{R}^{d}, \forall A \in \mathcal{A}, \mathbb{P}(A)=\mathbb{P}\left(\tau_{\mathbf{t}} A\right), \quad \tau_{\mathbf{t}} A=\left\{\omega, \tau_{-\mathbf{t}} \omega \in A\right\} .
$$

A real random variable, $X$, is a measurable map from $\Omega$ into $\mathbb{R}$. To each random variable $X$ corresponds a s.h. random process defined by:

$$
\forall \mathbf{t} \in \mathbb{R}^{d}, \forall \omega \in \Omega, \widetilde{X}(\omega, \mathbf{t})=X\left(\tau_{-\mathbf{t}} \omega\right)=\widetilde{X}\left(\tau_{-\mathbf{t}} \omega, 0\right)
$$

The set of real random variables $L^{p}(\Omega), 1 \leq p<+\infty$, is such that $\mathbb{E}|X|^{p}=$ $\int_{\omega \in \Omega}|X(\omega)|^{p} d \mathbb{P}<+\infty$. $\mathbb{E}$ is the ensemble average operator. The field $\mathbf{t} \rightarrow$ 
$\widetilde{X}(\omega, \mathbf{t})$ is almost surely (a.s.) in $L_{l o c}^{p}\left(\mathbb{R}^{d}\right)$ if $X \in L^{p}(\Omega)$. Here $L_{l o c}^{p}\left(\mathbb{R}^{d}\right)$ is the set of real functions $f$ such that $\int_{\mathbf{t} \in B}|f(\mathbf{t})|^{p} d \mathbf{t}<+\infty$ for any bounded open domain $B \subset \mathbb{R}^{d}$. Almost surely means for all $\omega$ in a subset of $\Omega$ with probability one. The following notations are used:

$$
\forall Y, Z \in L^{2}(\Omega),\langle Y Z\rangle(\mathbf{t})=\mathbb{E}(\widetilde{\delta Y}(\omega, 0) \widetilde{\delta Z}(\omega, \mathbf{t})), \quad \delta X=X-\mathbb{E} X
$$

Elasticity of the random medium is described with $\widetilde{\mathbf{c}}(\omega, \mathbf{t})$, the s.h. random field of stiffness tensor. For two-phase composites $\mathbf{c}(\omega)=\chi(\omega) \mathbf{c}^{I}+(1-\chi(\omega)) \mathbf{c}^{I I}$ where the characteristic function of phase $I$ is $\widetilde{\chi}(\omega, \mathbf{t})=\chi\left(\tau_{-\mathbf{t}} \omega\right)=1$ when $\mathbf{t}$ lies in phase $I$, and $=0$ otherwise. The $k$-point probability function of simultaneously finding $k$ points with positions $\mathbf{t}_{1}, \cdots, \mathbf{t}_{k}$ in phase $I$ is:

$$
S_{k}\left(\mathbf{t}_{1}, \cdots, \mathbf{t}_{k}\right)=\mathbb{E} \tilde{\chi}\left(\omega, \mathbf{t}_{1}\right) \cdots \widetilde{\chi}\left(\omega, \mathbf{t}_{k}\right)
$$

Note that $S_{k}\left(\mathbf{t}_{1}, \cdots, \mathbf{t}_{k}\right)=S_{k}\left(\mathbf{t}_{1}+\mathbf{t}, \cdots, \mathbf{t}_{k}+\mathbf{t}\right)$. In particular, $S_{1}$ is uniform and is equal to the volume fraction of phase $I$.

As an example, consider an infinite composite consisting of identical overlapping spherical inclusions of radius $R$ (diameter $D=2 R$ ) randomly embedded within a matrix (phase I). Then, a realization $\omega \in \Omega$ is the set $\omega=\left\{\mathbf{x}_{n}, n \in \mathbb{N}\right\}$ of the centres $\mathbf{x}_{n}$ of the inclusions, $\tau_{\mathbf{t}} \omega=\left\{\mathbf{x}_{n}+\mathbf{t}, n \in \mathbb{N}\right\}$ and $\chi(\omega)=1$ if $\operatorname{Inf}\left|\mathbf{x}_{n}\right|>R$, and $=0$ otherwise. In order to guarantee the statistical homogeneity of the composite, $\mathbb{P}$ must be invariant under the action of $\tau_{\mathbf{t}}$, for all t. This will be the case if $\mathbb{P}$ obeys the well-known Poisson distribution with number density $\rho>0$. For this distribution [7] and [8],

$$
S_{k}\left(\mathbf{t}_{1}, \cdots, \mathbf{t}_{k}\right)=\exp \left[-\rho v_{k, R}\left(\mathbf{t}_{1}, \cdots, \mathbf{t}_{k}\right)\right]
$$

where $v_{k, R}\left(\mathbf{t}_{1}, \cdots, \mathbf{t}_{k}\right)$ is the union volume of $k d$-dimensional spheres of radius $R$ centred at $\mathbf{t}_{1}, \cdots, \mathbf{t}_{k}$.

\section{Homogenization of s.h. random media}

Determination of the homogenized elasticity tensor, $\mathbf{C}^{\text {hom }}$, requires the resolution of the following problem [5], [6]:

$$
\boldsymbol{\sigma} \in \mathcal{S}, \mathbf{e}^{*} \in \mathcal{K}^{*}, \boldsymbol{\sigma}(\omega)=\mathbf{c}(\omega):\left(\mathbf{e}^{*}(\omega)+\mathbf{E}\right) \text { a.s., } \mathbb{E} \boldsymbol{\sigma}=\mathbf{C}^{\text {hom }}: \mathbf{E}
$$

where $\mathbf{E}$ is the macroscopic strain; $\mathcal{K}^{*}$, the set of kinematically compatible s.h. strain fields with null ensemble average, and $\mathcal{S}$, the set of statically compatible s.h. stress fields, are defined by: 


$$
\begin{aligned}
\mathcal{K}^{*} & =\left\{\mathbf{e}^{*}=\left(e_{i j}^{*}\right), e_{i j}^{*}=e_{j i}^{*} \in L^{2}(\Omega), \mathbb{E} e_{i j}^{*}=0, \widetilde{\mathbf{e}}^{*}(\omega, \mathbf{t}) \text { compatible a.s. }\right\} \\
\mathcal{S} & =\left\{\boldsymbol{\sigma}=\left(\sigma_{i j}\right), \sigma_{i j}=\sigma_{j i} \in L^{2}(\Omega), \operatorname{div} \widetilde{\boldsymbol{\sigma}}(\omega, \mathbf{t})=0 \text { a.s. }\right\}
\end{aligned}
$$

The compatibility of $\mathbf{t} \rightarrow \widetilde{\mathbf{e}}^{*}(\omega, \mathbf{t})$ and the divergence operator are in the usual sense of the distributions $\mathcal{D}^{\prime}\left(\mathbb{R}^{d}\right)$.

Introducing a homogeneous comparison medium having elasticity tensor $\mathbf{C}^{0}$, polarization tensor $\mathbf{p}=\boldsymbol{\sigma}-\mathbf{C}^{0}:\left(\mathbf{e}^{*}+\mathbf{E}\right)$ is solution to a Lippmann-Schwinger equation:

$$
\mathbf{p}=\left(\mathbf{c}-\mathbf{C}^{0}\right):\left(-\Gamma^{0} * \delta \mathbf{p}+\mathbf{E}\right)
$$

For $\mathbf{p}^{\prime}=\left(p_{i j}^{\prime}\right) p_{i j}^{\prime}=p_{j i}^{\prime} \in L^{2}(\Omega),\left(-\boldsymbol{\Gamma}^{0} * \mathbf{p}^{\prime}\right)$ is the unique solution to:

$$
\left(-\boldsymbol{\Gamma}^{0} * \mathbf{p}^{\prime}\right) \in \mathcal{K}^{*},\left(\mathbf{C}^{0}:\left(-\boldsymbol{\Gamma}^{0} * \mathbf{p}^{\prime}\right)+\mathbf{p}^{\prime}\right) \in \mathcal{S}
$$

Moreover, $\mathbf{p}$ makes stationary the following functional [9]:

$$
\mathcal{H}\left(\mathbf{p}^{\prime}\right)=\mathbb{E}\left(2 \mathbf{p}^{\prime}: \mathbf{E}-\mathbf{p}^{\prime}:\left(\mathbf{c}-\mathbf{C}^{0}\right)^{-1}: \mathbf{p}^{\prime}-\mathbf{p}^{\prime}: \Gamma^{0} * \mathbf{p}^{\prime}\right)
$$

And, $\mathbf{C}^{\text {hom }}$ can be expressed as follows:

$$
\mathbf{C}^{\text {hom }}: \mathbf{E}=\mathbb{E} \mathbf{c}: \mathbf{E}-\mathbb{E}\left(\delta \mathbf{c}: \Gamma^{0} * \delta \mathbf{p}\right)
$$

It will be assumed in the sequel that all correlation functions $\left\langle c_{i j m n} p_{k l}\right\rangle(\mathbf{t})$ are in $L^{2}\left(\mathbb{R}^{d}\right)$. Then, it can be proved that:

$$
\left[\mathbb{E}\left(\delta \mathbf{c}: \Gamma^{0} * \delta \mathbf{p}\right)\right]_{i j}=\int_{\mathbf{t} \in \mathbb{R}^{d}} \Gamma_{m n k l}^{0}(-\mathbf{t})\left\langle c_{i j m n} p_{k l}\right\rangle(\mathbf{t}) \mathrm{d} \mathbf{t}
$$

with $\Gamma_{m n k l}^{0}=\left(-G_{n k, m l}^{0}\right)^{\text {sym }}, \mathbf{G}^{0}(\mathbf{t})$ being the infinite-homogeneous-body Green's function for the comparison medium and the notation sym indicating symmetrization on subscripts $m n$ and $k l$. It should be emphasized that the value of (13) does not depend on $\mathbf{C}^{0}$, and that $-\mathbf{C}^{0}$ : $\mathbf{e}^{*}$ can be equivalently substituted for $\delta \mathbf{p}$ in (12) and (13).

\section{Periodization of random media}

For seek of simplicity, the analysis is restricted to two-phase composites. $V^{L}$ is the cube centred at the origin with sides of length $L>0$ parallel to the canonical basis of $\mathbb{R}^{d},\left\{\mathbf{e}_{1}, \cdots, \mathbf{e}_{d}\right\}$. The numerical computation of $\mathbf{C}^{\text {hom }}$ is often based on the generation of $L$-periodic samples which statistical properties on $V^{L}$ are very close to those of the considered medium. The subset $\Omega^{L} \subset \Omega$ of $L$-periodic realizations $\omega^{L} \in \Omega^{L}$ is introduced:

$$
\omega^{L} \in \Omega^{L} \Longleftrightarrow \tau_{\mathbf{u}} \omega^{L}=\omega^{L}, \forall \mathbf{u} \in\left\{L \mathbf{e}_{1}, \cdots, L \mathbf{e}_{d}\right\}
$$


Definition 1 A sequence of probability spaces $\left(\Omega^{L}, \mathcal{A}^{L}, \mathbb{P}^{L}\right)_{L>0}$ is a periodization of $(\Omega, \mathcal{A}, \mathbb{P})$ if $\tau_{\mathbf{t}}$ is a measure preserving flow on $\left(\Omega^{L}, \mathcal{A}^{L}, \mathbb{P}^{L}\right)$ (statistical insensitivity to translations) and, $\mathbb{E}^{L}$ being the ensemble average on $\Omega^{L}$,

$$
\begin{aligned}
\forall k, \forall \mathbf{t}_{1}, \cdots, \mathbf{t}_{k}, S_{k}^{L}\left(\mathbf{t}_{1}, \cdots, \mathbf{t}_{k}\right) & =\mathbb{E}^{L} \widetilde{\chi}\left(\omega^{L}, \mathbf{t}_{1}\right) \cdots \widetilde{\chi}\left(\omega^{L}, \mathbf{t}_{k}\right), \\
\lim _{L \rightarrow+\infty} S_{k}^{L}\left(\mathbf{t}_{1}, \cdots, \mathbf{t}_{k}\right) & =S_{k}\left(\mathbf{t}_{1}, \cdots, \mathbf{t}_{k}\right)
\end{aligned}
$$

Note that all realizations of a s.h. random process $\widetilde{X}\left(\omega^{L}, \mathbf{t}\right)$ of $\left(\Omega^{L}, \mathcal{A}^{L}, \mathbb{P}^{L}\right)$ are $L$-periodic; $\widetilde{\chi}\left(\omega^{L}, \mathbf{t}\right)$ is $L$-periodic and $S_{k}^{L}$ are also $L$-periodic in each $\mathbf{t}_{i}$. As a consequence, the overall elasticity tensor $\mathbf{C}^{L}\left(\omega^{L}\right)$ associated with each periodic realization $\omega^{L}$ can be classically obtained with standard periodic homogenization. Its ensemble average $\mathbf{C}^{\text {hom, } L}$ can be determined via equations (9) and (12) where the periodic operator $\boldsymbol{\Gamma}^{0, L}$ is substituted for $\boldsymbol{\Gamma}^{0}$ :

$$
\begin{aligned}
\mathbf{p}^{L}\left(\omega^{L}\right) & =\left(\mathbf{c}\left(\omega^{L}\right)-\mathbf{C}^{0}\right):\left(-\Gamma^{0, L} * \delta \mathbf{p}^{L}+\mathbf{E}\right) \\
\mathbf{C}^{\text {hom }, L} & : \quad \mathbf{E}=\mathbb{E}^{L} \mathbf{c}: \mathbf{E}-\int_{\mathbf{t} \in V^{L}} \Gamma_{m n k l}^{0, L}(-\mathbf{t})\left\langle c_{i j m n} p_{k l}^{L}\right\rangle(\mathbf{t}) \mathrm{d} \mathbf{t}
\end{aligned}
$$

Here, $\delta X\left(\omega^{L}\right)=X\left(\omega^{L}\right)-\mathbb{E}^{L} X$ and $\left\langle c_{i j m n} p_{k l}^{L}\right\rangle$ are the $L$-periodic correlation functions of the periodized medium. Introducing

$$
\left\langle c_{i j m n} p_{k l}^{L}\right\rangle^{\infty}(\mathbf{t})=\begin{array}{cl}
\left\langle c_{i j m n} p_{k l}^{L}\right\rangle(\mathbf{t}) & \forall \mathbf{t} \in V^{L} \\
0 & \forall \mathbf{t} \in \mathbb{R}^{d} \backslash V^{L}
\end{array}
$$

and using standard Fourier's analysis [11], equation (18) can be established.

$$
\int_{\mathbf{t} \in V^{L}} \Gamma_{m n k l}^{0, L}(-\mathbf{t})\left\langle c_{i j m n} p_{k l}^{L}\right\rangle(\mathbf{t}) \mathrm{d} \mathbf{t}=\int_{\mathbf{t} \in \mathbb{R}^{d}} \Gamma_{m n k l}^{0}(-\mathbf{t})\left\langle c_{i j m n} p_{k l}^{L}\right\rangle^{\infty}(\mathbf{t}) \mathrm{d} \mathbf{t}
$$

Comparing (12-13) and (16-18), the following convergences are expected:

$$
\begin{gathered}
\left\langle c_{i j m n} p_{k l}^{L}\right\rangle^{\infty}(\mathbf{t}) \underset{L \rightarrow+\infty}{\longrightarrow}\left\langle c_{i j m n} p_{k l}\right\rangle(\mathbf{t}) \text { in } L^{2}\left(\mathbb{R}^{d}\right) \\
\mathbf{C}^{\text {hom }, L} \underset{L \rightarrow+\infty}{\longrightarrow} \mathbf{C}^{\text {hom }}
\end{gathered}
$$

\section{Definition of RVE}

For an ideal periodization sequence $\left(\Omega^{L}, \mathcal{A}^{L}, \mathbb{P}^{L}\right)_{L>0}$ such that $\mathbb{E}^{L} \mathbf{c}=\mathbb{E} \mathbf{c}$ and $\left\langle c_{i j m n} p_{k l}^{L}\right\rangle^{\infty}(\mathbf{t})=\left\langle c_{i j m n} p_{k l}\right\rangle(\mathbf{t}), \forall \mathbf{t} \in V^{L}$, the error $\mathbf{C}^{\text {hom, } L}-\mathbf{C}^{\text {hom }}$ can be expressed in terms of correlations $\left\langle c_{i j m n} p_{k l}\right\rangle(\mathbf{t})$ for $\mathbf{t}$ outside $V^{L}$. This leads to the following definition.

Definition $2 V^{L}$ is an $\epsilon-R V E, \epsilon>0$, if (20) holds true. 


$$
\begin{aligned}
\exists \mathbf{C}^{0}, \forall \mathbf{E}, \forall L^{\prime} & \geq L, \\
\left\|\int_{\mathbf{t} \in \mathbb{R}^{d} \backslash V^{L^{\prime}}} \Gamma_{m n k l}^{0}(-\mathbf{t})\left\langle c_{i j m n} p_{k l}\right\rangle(\mathbf{t}) \mathrm{d} \mathbf{t}\right\| & \leq \epsilon\left\|\mathbf{C}^{\mathrm{hom}}: \mathbf{E}\right\|
\end{aligned}
$$

Here $\|\cdot\|$ is a norm on $\mathbb{R}^{d} \times \mathbb{R}^{d}$ and $\epsilon$ is the relative error on the overall elasticity tensor for an ideal periodization sequence. It should be emphasized here that for actual periodization sequences, $\left\langle c_{i j m n} p_{k l}^{L}\right\rangle^{\infty}$ does not coïncide exactly with $\left\langle c_{i j m n} p_{k l}\right\rangle$ on $V^{L}$. Therefore, the rate of convergence of $\mathbf{C}^{\text {hom, } L}$ to $\mathbf{C}^{\text {hom }}$ depends on the adopted periodization sequence and convergence up to a given relative error $\epsilon$ may occur for unit cell size larger than the minimum $\epsilon$-RVE size.

Note that the use of homogeneous strain or stress boundary conditions introduces a systematic bias in the estimation of the overall elasticity tensor because the corresponding $\Gamma$ operator does not preserve the statistical homogeneity of the medium. See also [12] and [13].

In $(20),\left\langle c_{i j m n} p_{k l}\right\rangle$ can be formally expressed in terms of $\mathbf{C}^{0}, \mathbf{c}^{I}, \mathbf{c}^{I I}$ and the set of all probability functions $S_{k}$ as shown in [10]. Following H-S-W method used in [4], an estimation for the minimum RVE size based on the two-point probability function can be obtained as follows. The stationarity of functional (11) is restricted to piecewise-uniform polarization tensors on each phase, $\mathbf{p}^{H S W}(\omega)=\chi(\omega) \mathbf{p}^{I}+(1-\chi(\omega)) \mathbf{p}^{I I}$, where $\mathbf{p}^{I}$, $\mathbf{p}^{I I}$ and correlation functions $\left\langle c_{i j m n} p_{k l}^{H S W}\right\rangle$ are determined in terms of $\mathbf{C}^{0}, \mathbf{c}^{I}, \mathbf{c}^{I I}, S_{1}$ and $S_{2}$. Substituting $\left\langle c_{i j m n} p_{k l}^{H S W}\right\rangle$ for $\left\langle c_{i j m n} p_{k l}\right\rangle$ in (20), one can estimate the minimum RVE size in terms of $\mathbf{C}^{0}, \mathbf{c}^{I}, \mathbf{c}^{I I}, S_{1}$ and $S_{2}$. In the special case of the above described inclusions-matrix composite, $\left\langle c_{i j m n} p_{k l}^{H S W}\right\rangle=0$ for $|\mathbf{t}| \geq D$ can be easily derived from (6). It follows from the H-S-W approximation that $2 D$ is an estimation for the minimum RVE size, for any phase contrast and any relative error $\epsilon$, which is in agreement with [4].

\section{Numerical study}

A $L$-periodized sample of the above described inclusions-matrix composite is generated as follows: first, the number $N$ of inclusions centres in $V^{L}$ is generated according to the Poisson integer random variable with parameter $\rho L^{d}$. Then, $N$ centres are uniformly distributed in $V^{L}$ and they are extended to the whole space by $L$-periodicity. It can be proved that the $L$-periodized medium is statistically invariant with respect to translations and that the corresponding $k$-point matrix probability function is given by:

$$
S_{k}^{L}\left(\mathbf{t}_{1}, \cdots, \mathbf{t}_{k}\right)=\exp \left[-\rho v_{k, R}^{L}\left(\mathbf{t}_{1}, \cdots, \mathbf{t}_{k}\right)\right]
$$

where $v_{k, R}^{L}\left(\mathbf{t}_{1}, \cdots, \mathbf{t}_{k}\right)$ is the union volume in $V^{L}$ of the $d$-dimensional spheres of radius $R$ centred at $\mathbf{t}_{1}, \cdots, \mathbf{t}_{k}$ and all their $L$-periodic images $\mathbf{t}_{1}+L \mathbf{z}_{1}, \cdots, \mathbf{t}_{k}+$ $L \mathbf{z}_{k}, \mathbf{z}_{1}, \cdots, \mathbf{z}_{k} \in \mathbb{Z}^{d}$. The following relationships can be established: 


$$
\begin{array}{r}
\forall L \geq D, \forall k \geq 1, \forall \mathbf{t}_{1}, \cdots, \mathbf{t}_{k} \in V^{L-D}, \quad S_{k}^{L}\left(\mathbf{t}_{1}, \cdots, \mathbf{t}_{k}\right)=S_{k}\left(\mathbf{t}_{1}, \cdots, \mathbf{t}_{k}\right) \\
\forall L \geq D, \forall \mathbf{t}, \quad S_{1}^{L}(\mathbf{t})=S_{1}(\mathbf{t})=S_{1}(\mathbf{0}), \\
\forall L \geq 2 D, \forall \mathbf{t} \in V^{L}, \forall \mathbf{t}_{1}, \quad S_{2}^{L}\left(\mathbf{t}_{1}, \mathbf{t}_{1}+\mathbf{t}\right)=S_{2}\left(\mathbf{t}_{1}, \mathbf{t}_{1}+\mathbf{t}\right)=S_{2}(\mathbf{0}, \mathbf{t}) .
\end{array}
$$

Note that, for all $L \geq 2 D$, the one- and two-point probability functions of the $L$-periodized medium coincide on $V^{L}$ with those of the original medium.

The convergence of $\mathbf{C}^{\text {hom, } L}$ to $\mathbf{C}^{\text {hom }}$ for increasing $L$ can be proved using the results of [13]. In order to illustrate the theoretical analysis of the previous sections, 2D finite element computations have been conducted on the periodized material with CESAR-LCPC software and $\left\langle c_{i j m n} e_{k l}^{L}\right\rangle$ have been plotted. Square elements (Q4) have been used (length $\left./ D=\frac{1}{16} \sqrt{\frac{\pi}{2}}\right)$. Isotropic phases with the same Poisson's ratio (0.3) and two different shear modulus contrast $G^{\text {inclusion }} / G^{\text {matrix }}$ (10 and 100 ) have been considered. Several ratios $L / D$ $(1.253,2.507,5.013$ and 10.026$)$ corresponding to $16 \times 16,32 \times 32,64 \times 64$ and $128 \times 128$ elements have been considered. Using statistical homogeneity and periodicity, the computation of correlation function $\langle X Y\rangle$ on the periodized medium is conducted as follows: $n$ periodic realizations $\omega_{i}^{L}$ are generated; for each realization, the spatial correlation function $\frac{1}{\left|V^{L}\right|} \int_{\mathbf{u} \in V^{L}} \widetilde{\delta X}\left(\omega_{i}^{L}, \mathbf{u}\right) \widetilde{\delta Y}\left(\omega_{i}^{L}, \mathbf{t}+\mathbf{u}\right) \mathrm{d} \mathbf{u}$ is computed using FFT techniques; and $\langle X Y\rangle$ is obtained by (ensemble) averaging spatial correlation functions. The normalized average overall shear modulus $\bar{G}$ and its standard deviation $s$ are reported in Table 1 for $S_{1}=0.5$. The number of realizations $n$ is such that $1,96 \frac{s}{\sqrt{n} \bar{G}} \leq 0.02$. Numerical estimates for $S_{1}$ (ensemble average of matrix volume fraction) are also provided. Note that the convergence is slower for phase contrast 100. In this case, the overall shear modulus is twice the Hashin-Shtrikman lower bound and one fifth the upper bound. As shown in figure 1, the normalized correlation function $\left\langle\chi e_{12}^{L}\right\rangle$ for $L / D=10.026$, is very small outside a circular domain of radius $D$. Similar result is obtained for phase contrast 10 . This plaids for a minimum RVE size equal to $2 D$ in agreement with the two-probability function estimation. On the other hand, it is clear from Table $1 \mathrm{~b}$ and figure 2, where a diagonal profile of figure 1 is shown for three ratios $L / D$, that the predictions of the periodized material are not accurate for $L / D=2.507$. These predictions become accurate when the plateau appears for $L / D=5.013$. So, $2 D$ is the minimum RVE size for any phase contrast while the convergence of $\mathbf{C}^{\text {hom,L }}$ to $\mathbf{C}^{\text {hom }}$ for high phase contrast occurs for cell size larger than $2 D$.

The smallest RVE is defined in this Note as the "support domain" of correlation functions $\left\langle c_{i j m n} e_{k l}\right\rangle$ or $\left\langle c_{i j m n} p_{k l}\right\rangle$. Using H-S-W approximation method, this definition allows for simple determination of minimum RVE size in agreement with [4]. Numerical simulations show that the convergence of $\mathbf{C}^{\text {hom, } L}$ to $\mathbf{C}^{\text {hom }}$ occurs when $\delta \mathbf{c}(0)$ and $\delta \mathbf{p}^{L}(\mathbf{t})$ become uncorrelated for $\mathbf{t}$ near the boundary of the unit cell $V^{L}$. For high contrast phase, the convergence occurs for unit cell size of the periodized material larger than the minimum RVE size. Studying 
convergence (19) and finding a quantitative estimation of $\mathbf{C}^{\text {hom, } L}-\mathbf{C}^{\text {hom }}$ based on the correlation functions of the periodized medium are interesting issues for future work.

\section{REFERENCES}

[1] A. A. Gusev, Representative volume element size for elastic composites: a numerical study, J. Mech. Phys. Solids 45 (1997) 1449-1459.

[2] K. Terada, T. Ito, N. Kikuchi, Characterization of the mechanical behaviors of solid-fluid mixture by the homogenization method, Comput. Methods Appl. Engng, 153 (1998) 223-257.

[3] T. Kanit, S. Forest, I. Galliet, V. Mounoury, D. Jeulin, Determination of the size of the representative volume element for random composites: statistical and numerical approach, Int. J. Solids Struct. 40 (2003) 3647-3679.

[4] W. J. Drugan, J. R. Willis, A micromechanics-based nonlocal constitutuve equation and estimates of representative volume element size for elastic composites, J. Mech. Phys. Solids 44 (4) (1996) 497-524.

[5] K. Sab, Principe de Hill et homogénéisation des matériaux aléatoires, C. R. Acad. Sci Paris, Sér II 312 (1991) 1-5.

[6] K. Sab, Homogenization of non-linear random media by a duality method. Application to plasticity, Asymp. Anal. 9 (1994) 311-336.

[7] G. Matheron, Eléments pour une théorie des milieux poreux, Masson, Paris. (1967).

[8] S. Torquato, Random heterogeneous media: microstructure and improved bounds on effective properties, Appl. Mech. Rev. 44 (1991) 37-76.

[9] J. R. Willis, Bounds and self-consistent estimates for the overall properties of anisotropic composites, J. Mech. Phys. Solids 25 (1977) 185-202.

[10] E. Kröner, Linear properties of random media - the systemtic theory, in Comportements rhéologiques et structure des matériaux, CR 15ème Coll. GFR. Paris. (1980).

[11] P. Suquet, Une méthode simplifiée pour le calcul des propriétés élastiques de matériaux hétérogènes à structure périodique, C. R. Acad. Sci Paris, Sér II 311 (1990) 769-774.

[12] C. Huet, Application of variational concepts to size effects in elastic heterogeneous bodies, J. Mech. Phys. Solids 38 (1990) 813-841.

[13] K. Sab, On the homogenization and the simulation of random materials, Eur. J. Mech. A/Solids, 11 (1992) 585-607. 


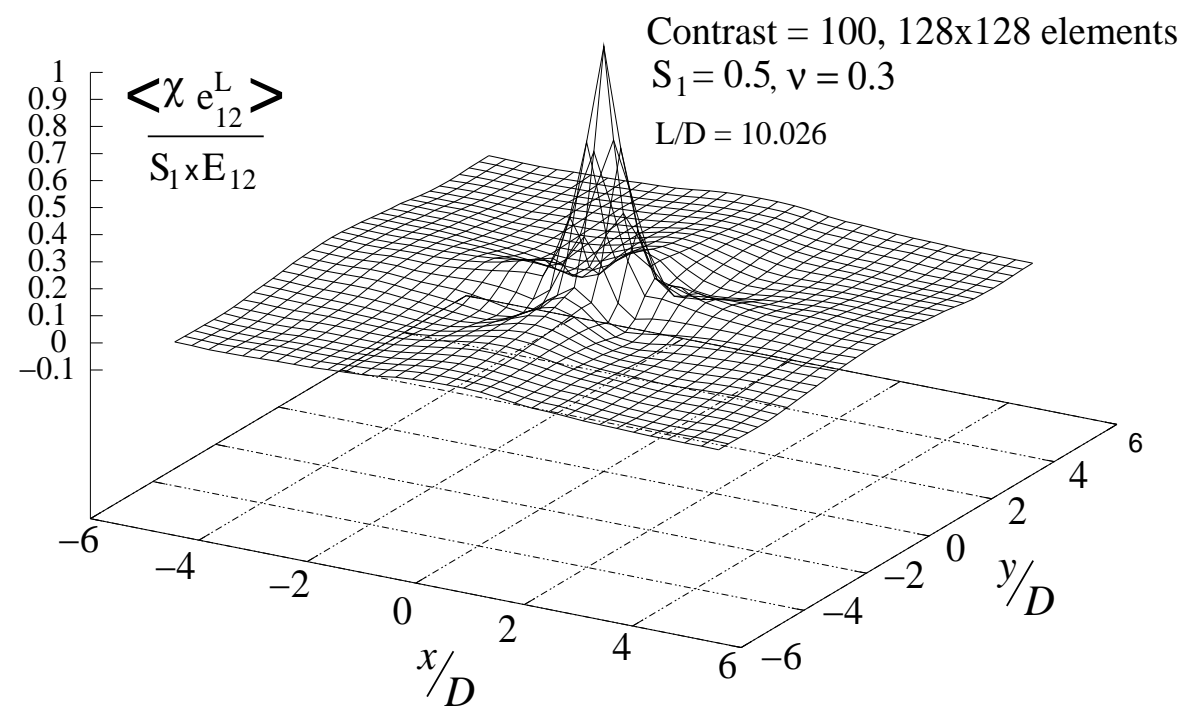

FIG. 1 - Normalized correlation function $\left\langle\chi e_{12}^{L}\right\rangle$.

Figure 1 - Fonction de corrélation $\left\langle\chi e_{12}^{L}\right\rangle$ normalisée. 


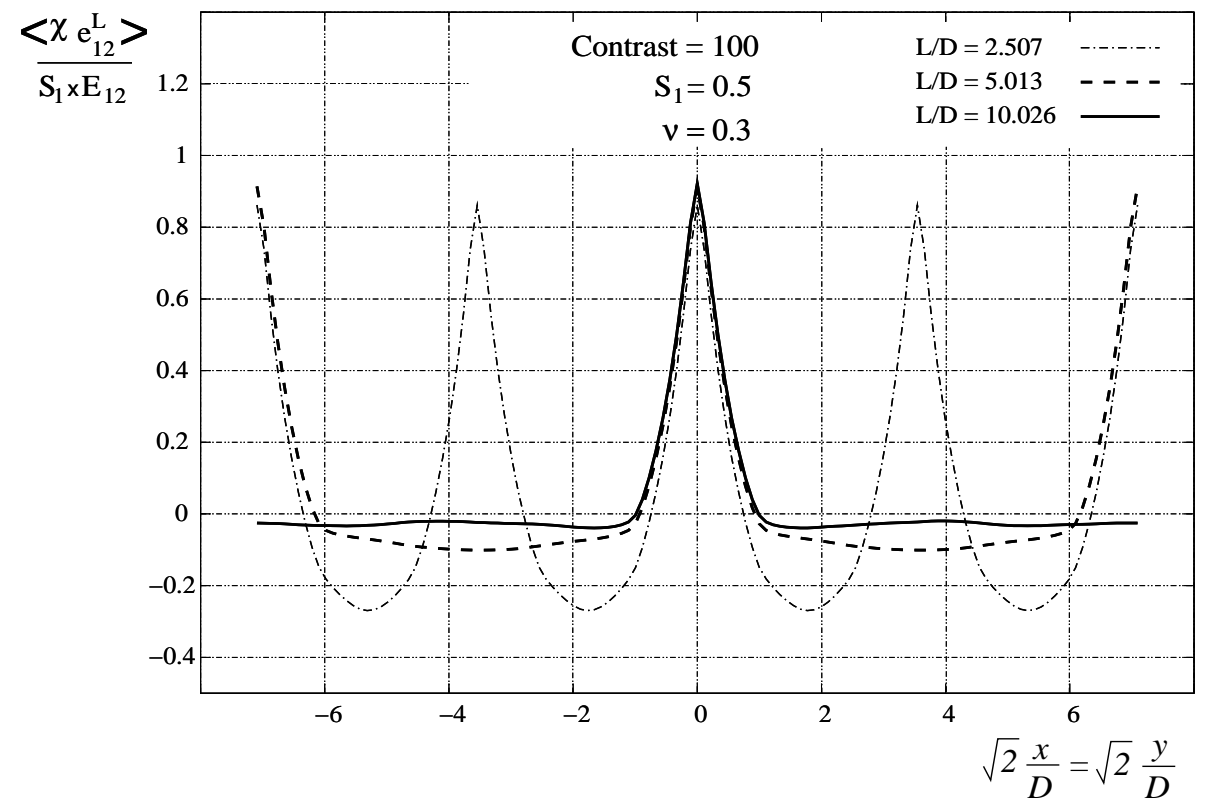

FIG. 2 - Diagonal profile of normalized $\left\langle\chi e_{12}^{L}\right\rangle$ for different $L / D$ ratios.

Figure 2 - Profil diagonal de la fonction $\left\langle\chi e_{12}^{L}\right\rangle$ normalisée pour différents rapports $L / D$. 
(a)

\begin{tabular}{||l|l|l|l|l||}
\hline$L / D$ ratio & 1.253 & 2.507 & 5.013 & 10.026 \\
\hline Number of samples & 5706 & 1820 & 404 & 124 \\
\hline Mean volume fraction $S_{1}$ & 0.5023 & 0.4991 & 0.5024 & 0.4962 \\
\hline \hline Normalized shear modulus $\bar{G}$ & 3.3660 & 2.7922 & 2.6401 & 2.6598 \\
\hline$\sigma_{\bar{G}}$ & 2.5943 & 1.2154 & 0.5402 & 0.3006 \\
\hline
\end{tabular}

(b)

\begin{tabular}{||l|l|l|l|l||}
\hline$L / D$ ratio & 1.253 & 2.507 & 5.013 & 10.026 \\
\hline Number of samples & 25112 & 16440 & 3032 & 642 \\
\hline Mean volume fraction $S_{1}$ & 0.4977 & 0.4995 & 0.5004 & 0.5009 \\
\hline \hline Normalized shear modulus $\bar{G}$ & 17.4944 & 7.6908 & 5.1404 & 4.7527 \\
\hline$\sigma_{\bar{G}}$ & 28.2881 & 10.0622 & 2.8870 & 1.2276 \\
\hline
\end{tabular}

TAB. 1 - Numerical results for overall shear modulus, $\nu=0.3, S_{1}=0.5$. (a) Contrast $=10$, (b) Contrast $=100$.

Tableau 1 - Résultats numériques pour le module de cisaillement, $\nu=0.3, S_{1}=0.5$. (a) Contraste $=10$, (b) Contraste $=100$. 